\title{
Novel Integrated Sources for Short-reach Transmission Over Dispersive Channel
}

\author{
C Peucheret, ${ }^{1}$ M. E. Chaibi, ${ }^{1,2}$ L. Bramerie, ${ }^{1}$ K. Hassan, ${ }^{3}$ M. Gay, ${ }^{1}$ and D. Erasme ${ }^{4}$ \\ ${ }^{1}$ Univ Rennes, CNRS, FOTON - UMR 6082, F-22305 Lannion, France \\ ${ }^{2}$ Centre de Nanosciences et de Nanotechnologies, CNRS, Univ. Paris-Sud, Université Paris-Saclay, C2N-Orsay, F-91405 Orsay, France \\ ${ }^{3}$ University Grenoble Alpes, CEA, LETI, MINATEC Campus, F-38054 Grenoble, France \\ ${ }^{4}$ LTCI, Telecom ParisTech, Paris-Saclay University, F-75013 Paris, France \\ christophe.peucheret@univ-rennes1.fr
}

\begin{abstract}
We review our recent results on $\mathrm{Si}$ or III-V integrated devices in novel transmitter architectures for IM-DD dispersion-uncompensated links. Methods relying on chirp engineering or SSB generation are demonstrated for multi- and single-carrier modulation formats.

OCIS codes: (060.2360) Fiber optics links and subsystems; (060.4080) Modulation; (130.4110) Modulators; (140.5960) Semiconductor lasers.
\end{abstract}

\begin{abstract}
1. Introduction
Optical transmission systems employing intensity modulation (IM) and direct detection (DD) have recently been the object of renewed interest following the development of data-center interconnects (DCIs), where considerations of cost, footprint, and energy efficiency are critical. While photonic integration should be able to fulfil these requirements, new constraints are being introduced as a result of the evolution of data center networks towards more distributed architectures, with target link lengths of $10-120 \mathrm{~km}$. In this segment, the used of dense wavelengthdivision multiplexing (DWDM) in the C-band is being preferred due to the availability of legacy components [1]. The optical links therefore become dispersion-limited and proper mitigation techniques need to be implemented in order to avoid the use of costly and bulky optical dispersion compensation modules (DCMs). In some scenarios, the inherent latency of some digital signal processing (DSP) functionalities, including some forward error correction (FEC) schemes, also needs to be avoided.

Over the past few years, several technical solutions have been proposed and demonstrated for such dispersionlimited links employing IM and DD, fueling the debate between proponents of multi-carrier solutions employing orthogonal frequency division multiplexing (OFDM) and single-carrier approaches, typically making use of 4-level pulse amplitude modulation (PAM-4). Our work contributes to this on-going debate by proposing and experimentally demonstrating new approaches for transmission over a dispersive fiber link using either OFDM or PAM-4 modulation. The proposed techniques either rely on single-sideband (SSB) modulation, or on directly- or externally-modulated laser frequency chirping properties. In particular, we show that the effect of fading dips, which are inherent to DD systems, can be circumvented by optimized SSB filtering of a directly-modulated OFDM signal using silicon micro-ring resonators (MRRs) in series [2]. We also propose a novel transmitter structure exploiting the frequency chirping properties of two ring resonator modulators (RRMs) in series that have opposite frequency detunings with respect to a continuous wave $(\mathrm{CW})$ laser frequency [3]. Finally, we demonstrate the first generation of PAM-4 SSB signals using a dual electro-absorption modulated laser (D-EML) [4].
\end{abstract}

\section{Multi-carrier transmission}

For analogue modulation, a well know-effect of dispersion is the existence of frequency-dips in the small-signal frequency response of the channel, measured from the modulator (or directly-modulated laser - DML) driving signal input to the photodiode output, when double sideband (DSB) modulation is used. This effect, which limits the usable bandwidth when OFDM is employed, is obviously not present for SSB modulation. Optical filtering by MRRs in series has been shown to offer a good compromise between sideband suppression ratio and required guard-band in order to suppress one of the modulated sidebands while preserving the carrier. It was shown in [2] that a series of two silicon MRRs was sufficient to efficiently suppress one of the sidebands of a 14.7-Gbps OFDM signal made from 111 out of 128 subcarriers modulated with 8-state quadrature amplitude modulation (QAM), and transmit it over $100-\mathrm{km}$ standard single-mode fiber (SSMF), as illustrated in Fig. 1. Thanks to the tremendous progress of heterogeneous integration of III-V semiconductor devices on silicon, for instance through die or wafer bonding, the scheme is furthermore potentially integrable.

An alternative approach, where the passive MRRs need to be substituted with carrier depletion-based RRMs, is illustrated in Fig. 2(a) [3]. It relies on the observation that, under small signal modulation, moving from a positive to a negative frequency detuning $(\Delta \omega)$ of the RRM resonance with respect to the frequency of the CW laser induces a 

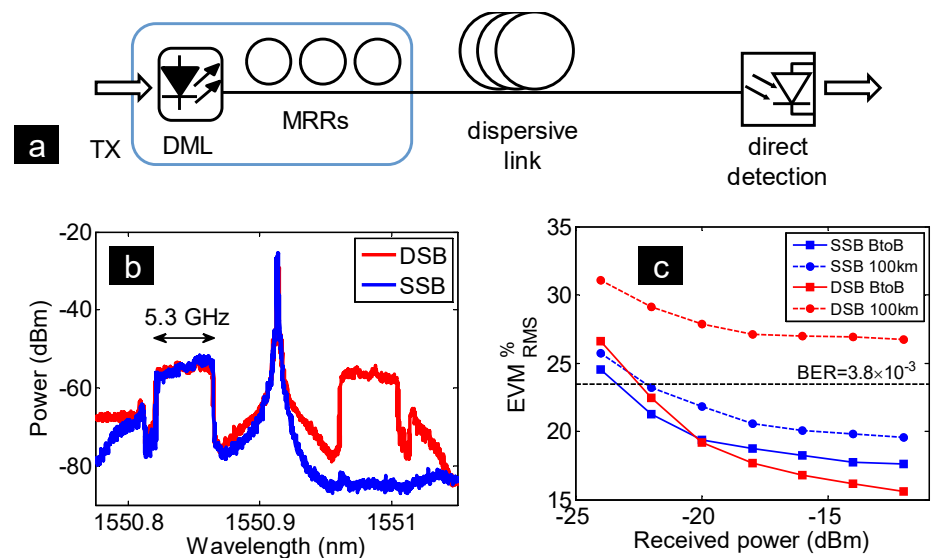

Fig. 2 (a) Principle of OFDM SSB signal transmission using a DML and passive MRRs in series. (b) Optical spectrum of DSB and SSB OFDM signals at $14.7 \mathrm{Gbps}$. (c) EVM performance for SSB and DSB signals back-to-back as well as after 100-km SSMF.

$\sim \pi$ jump between the intensity modulation and the chirp-induced phase modulation. This, in turn, affects the smallsignal frequency responses of the channel, which are different for $\pm \Delta \omega$ detunings. An interesting configuration is when two complementary frequency responses exist, as shown in Fig. 2(b) for a 100-km SSMF link. In this case, the subcarriers of the OFDM signal can be separated into two groups of sub-bands, each group being applied to one single RRM according to its individual frequency response. An example of such distribution based on the errorvector magnitude (EVM) of the individual subcarriers is represented in Fig. 2(c) for quadrature phase-shift keying (QPSK) modulation at an overall bit rate of $15 \mathrm{Gbps}$ (250 modulated subcarriers out of 256). Here 97\% of the subcarriers have EVM values below $-8.35 \mathrm{~dB}$, corresponding to a bit-error-ratio (BER) of $4.45 \times 10^{-3}$. EVMs averaged over all subcarriers are represented in Fig. 2(d) in back-to-back configuration, after 100-km SSMF when all subcarriers are applied to a single RRM with either positive or negative detuning, as well as when the subcarriers are distributed between the two RRMs. The graph clearly illustrates the potential of the scheme where distributing the subcarriers between the two RRMs results in performance close to back-to-back, and below the FEC threshold.
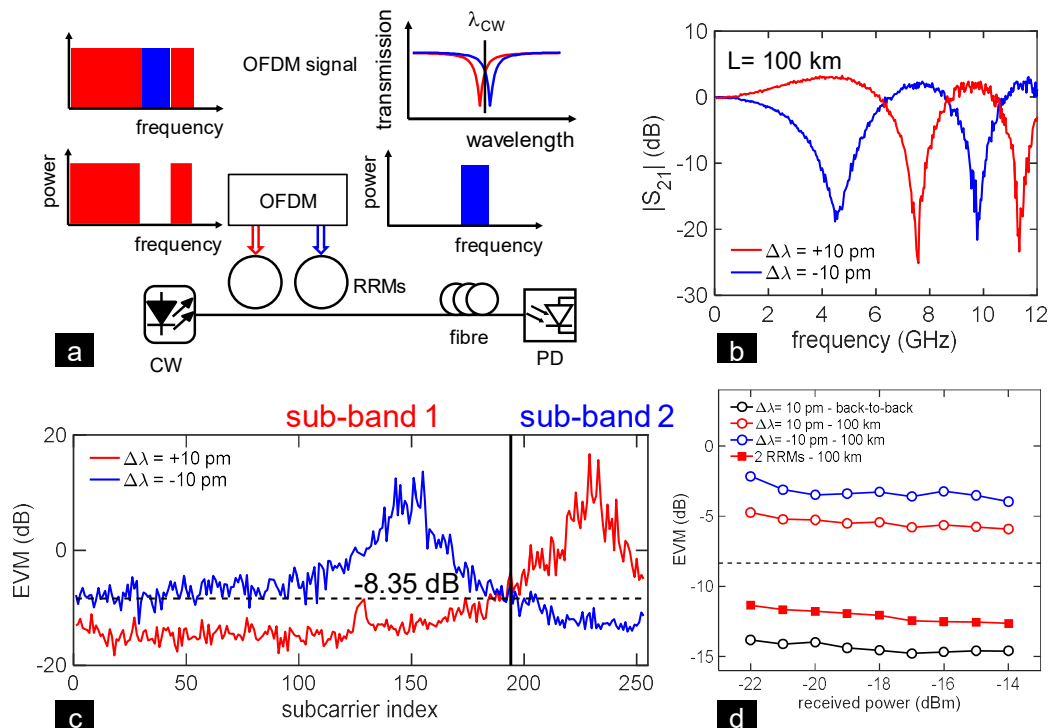

Fig. 2 (a) Principle of OFDM signal generation by band splitting and external modulation with two symmetrically-detuned RRMs (b) Channel response after 100-km SSMF for both positive and frequency detuning of the RRM resonance. (c) Corresponding EVM as a function of subcarrier index when either positive or negative detuning is used. (d) BER performance.

\section{Single-carrier transmission}

The generation of PAM-4 SSB signals has also recently been given consideration, essentially using complex inphase and quadrature or dual-drive Mach-Zehnder modulators. In [4], we have demonstrated PAM-2 and PAM-4 SSB signal generation relying on joint frequency modulation (FM) and intensity modulation (IM) in the DFB and electro-absorption modulation (EAM) sections, respectively, of a D-EML. Indeed, it can be shown that an SSB 
signal can be obtained when a proper relation between the IM and FM modulation indices is satisfied, as well as with a proper phase shift, at all frequencies, between the IM and FM driving signals [5]. The implementation is however challenging for broadband modulation. We used a monolithically integrated D-EML (Fig. 3(a)) [6] together with a simple pre-emphasis scheme to compensate for the residual IM introduced by the DFB section and correct its frequency response. The resulting SSB spectrum is shown in Fig. 3(b) for PAM-2 modulation at 10.7 Gbps. Figure 3(c) shows the results of BER measurements for PAM-4 at 10.7 Gbd in back-to-back as well as after 20-km and 50-km SSMF for both DSB and SSB modulation, respectively. DSB modulation outperforms SSB modulation in the back-to-back configuration with about 5-dB power penalty at a BER of $10^{-9}$, which is linked to an extinction ratio degradation that is inherent to the pre-emphasis technique. After 20-km SSMF propagation, the BER performance of the DSB signal is dramatically degraded with the occurrence of BER floors at a BER level of $10^{-4}$. On the other hand, SSB signals provide acceptable performance even after 50-km SSMF with the occurrence of BER floors at a level as low as $10^{-7}$. The eye diagrams of Fig. 3(a)-(f) confirm the better resilience of PAM-4 SSB to the accumulated dispersion.

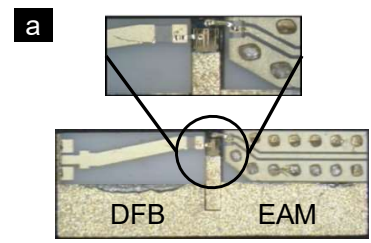

b
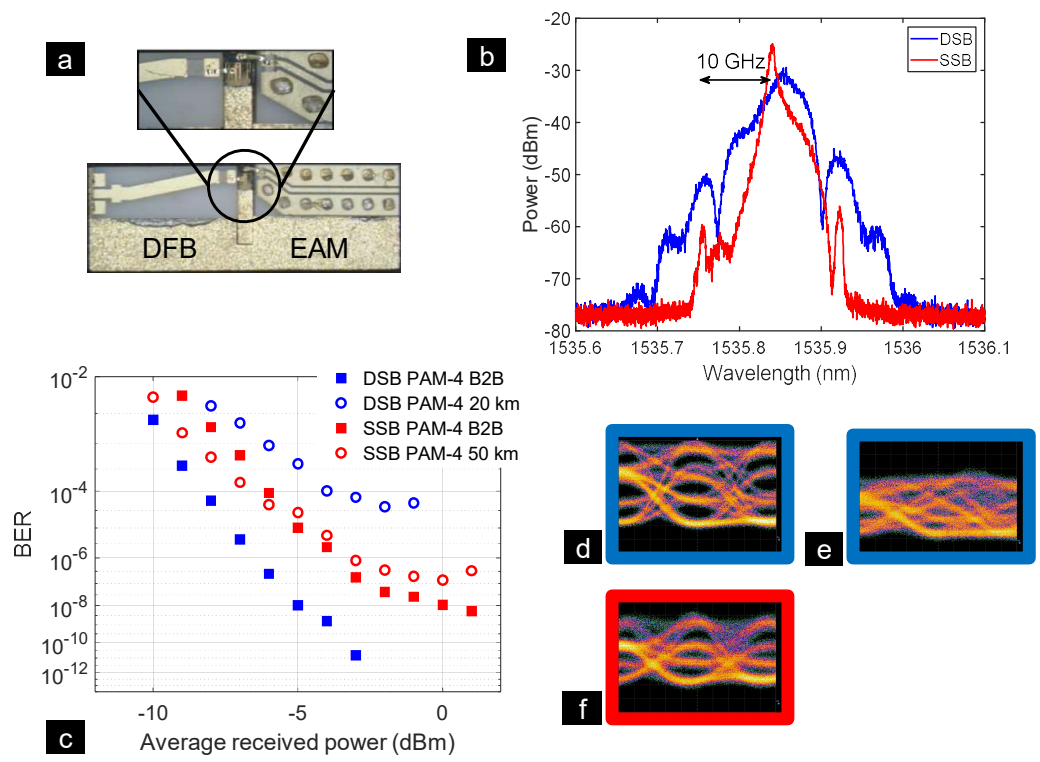

Fig. 3 (a) Monolithically integrated D-EML (III-V Lab). (b) Optical spectrum of a PAM-2 DSB signal generated by applying 10.7 Gbps data to the electro-absorption section of the D-EML. (c) Comparison of BER curves for PAM-4 DSB and SSB signals in back-to-back configuration and after transmission over SSMF. Corresponding eye diagrams for PAM-4 (d) DSB back-to-back, (e) DSB after 20-km and (f) SSB after 50-km.

\section{Conclusion}

The use of new integrated transmitter architectures for dispersion-tolerant transmission of single- or multi-carrier modulation in the C-band has been presented. SSB OFDM signals have been generated thanks to optimized filtering with MRRs in series, while a monolithically integrated D-EML has been used to generate SSB PAM-4. A new architecture exploiting the frequency chirping behavior of RRMs has also been presented for multicarrier modulation. All these schemes are potentially integrable and scalable in bandwidth.

\section{References}

[1] R. Nagarajan, M. Filer, Y. Fu, M. Kato, T. Rope, and J. Stewart, "Silicon photonics-based 100 Gbit/s, PAM4, DWDM data center interconnects," J. Opt. Commun. Netw. 10, B25-B36 (2018).

[2] M. Chaibi, K. Hassan, L. Bramerie, and C. Peucheret, "Directly-modulated IM/DD OFDM transmission over 100-km SSMF using SSB filtering with two silicon micro-ring resonators," in Conference on Lasers and Electro-Optics, OSA Technical Digest (Optical Society of America, 2017), paper STu1M.4.

[3] M. E. Chaibi, O. Dubray, L. Bramerie, K. Hassan, and C. Peucheret, "Multiple-band OFDM transmission exploiting the frequency chirping properties of silicon ring-resonator modulators," in Asia Communications and Photonics Conference, OSA Technical Digest (Optical Society of America, 2017), paper M2G.1.

[4] M. E. Chaibi, L. Bramerie, D. Erasme, and C. Peucheret, "Dispersion-uncompensated transmission of NRZ and PAM-4 single-sideband signals using D-EML," in Optical Fiber Communication Conference, OSA Technical Digest (Optical Society of America, 2018), paper Tu2C.3.

[5] H. Kim, "EML-based optical single sideband transmitter," IEEE Photon. Technol. Lett. 20, 243-245 (2008).

[6] D. Erasme, T. Anfray, M. E. Chaibi, K. Kechaou, J. Petit, G. Aubin, K. Merghem, C. Kazmierski, J.-G. Provost, P. Chanclou, and C. Aupetit-Berthelemot, "The dual-electroabsorption modulated laser, a flexible solution for amplified and dispersion uncompensated networks over standard fiber," J. Lightw. Technol. 32, 4068-4078 (2014). 